\section{Einfluss des Tetrachlormethans auf die Leber- struktur des Hundes in der Frühontogenese}

Unsere Experimente verfolgten den Einfluss des Tetrachlormethans $\left(\mathrm{CCl}_{4}\right)$ auf die morphologischen und biochemischen Veränderungen der Leber bei Hunden ${ }^{1-7}$. Wir benützten mit Mischkost ernährte Tiere mit einem Körpergewicht von $10-25 \mathrm{~kg}$, ohne Berücksichtigung von Rasse und Geschlecht. Das $\mathrm{CCl}_{4}$ wurde in regelmässigen Zeitspannen in einer Dosis von $0,5 \mathrm{bzw}, 0,3 \mathrm{ml} / \mathrm{kg}$ Körpergewicht verabreicht. Die Anordnung der Experimente wurde variiert.

Durch wiederholte Verabreichung von $\mathrm{CCl}_{4}$ gelang es uns, bei einer Gruppe der Tiere in 6 Monaten eine Lebercirrhose hervorzurufen 8,8 . Die morphologischen Veränderungen blieben meistens stationär oder nahmen zu, und nur in wenigen Fällen wurde eine Regression des cirrhotischen Prozesses beobachtet ${ }^{10}$. Durch fortgesetzte Applikation von $\mathrm{CCl}_{4}$ sollte festgestellt werden, wie die beschädigte Leber auf eine neue Zufuhr von hepatotoxischer Substanz reagiert und ob ein wesentlicher Unterschied zwischen der Reaktion einer normalen und beschädigten Leber besteht"1.

Eine Hündin bekam zu Ende der Versuchszeit, im Verlaufe der wiederholten und einige Monate dauernden $\mathrm{Zu}-$ fuhr von $\mathrm{CCl}_{4}, 5$ Junge. Nun interessierte uns der Einfluss der Gravidität auf die durch $\mathrm{CCl}_{4}$ geschädigte Leber, besonders aber, ob wiederholte Anwendung von $\mathrm{CCl}_{4}$ beim

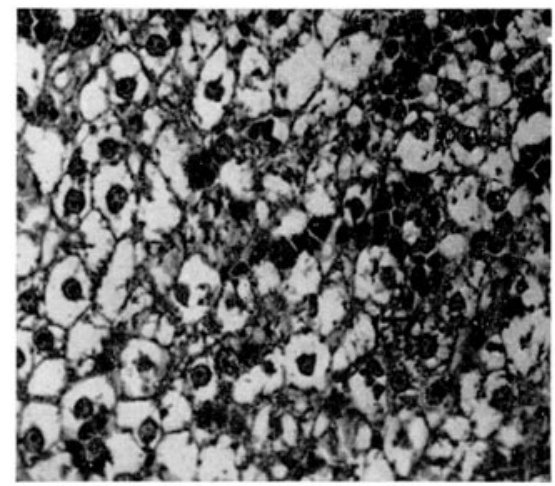

Diffuse, kleintröpfige Stea tose. In den Sinusoiden Herde einer extramedullären Hämatopoesis (HE, Vergr. 1:400).
Hund strukturelle Veränderungen der Leber in der frühen Ontogenese verursacht.

Evgebnisse. Der histologische Leberbefund der graviden Hündin war gleich wie bei den anderen Versuchstieren. Eine diffuse Fibrose mit nässender, grosströpfiger Steatose konnte festgestellt werden. Vereinzelt waren Pseudoläppchen, von groben Bindegewebsstreifen umgeben, sichtbar. Der histologische Befund aller 5 jungen Hunde ergab eine extramedulläre Hämatopoesis. Bei 3 jungen Hunden wurde eine nässende diffuse, kleintröpfige Steatose beobachtet; bei 2 jungen Hunden waren zonale, periportal lokalisierte Nekrosen vorhanden (Figur). Es waren weder eine Deformation noch Anzeichen fibrotischer oder cirrhotischer Veränderungen der Lober festzustellen.

Summary. By repeated administration of tetrachloromethane, cirrhosis of the liver was cvoked in dogs. In the course of the experiment one bitch had five litters, in all of which lesions of hepatic cells were found. In one group of animals, a diffuse steatosis of the liver was present; in the other group zonal necrosis was found. Malformations or fibrotic changes were not observed.

I. VIDo und F. Tomík

III. Medizinische Klinik der Komensky-Universität, Bratislava, und Pathologisch-anatomische Abteilung des Bezirksspitals, Trnava (Tschechoslowakei), 14. September 1964.

1 ]. DoBis, I. Vhuo und T. R. Nenerland, Cs. Gastruent. Výz. 15 255 (1961).

2 1. Vino, F. Tomik, J. Vtoo und J. Dobiš, Cs. Gastroent. Výz, 15 , $408(1961)$.

${ }^{3}$ J. DoBiš, I. Vino, J. Vino und T. R. Nienerland, Bratisl lek. Listy $41,537(1961)$.

4. VIDo, J. Vido und J. Dotrs, Bratisl. lek. Listy 42, 208 (1962),

3 I. Vido und T. R. Nrenertand, Acta hepato-splenol. 10, 182 (1963).

B. VIDo und T. R. NIEderland, Z. ges. Inn. Med. 18, 288 (1963).

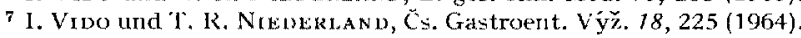

* I. Vido und T, R. Niederland, Acta hepato-splenol. 10, 361 (1963).

9 I. Vido, T. R. Niederland und F. Tomik, Rew. Czcch. Med. 9 245 (1963).

10 I. VIDO und F. Tomík, Triancel, in press.

11 I. Vido und J. Vino, Bratisl. jek. Listy, in press.

\section{Histologische Veränderungen der Magenschleimhaut des Hundes bei wiederholter Tetrachlormethanzufuhr}

Bekamntlich sind Magenstörungen im Verlaufe von Lebererkrankungen häufig. In der Humanmedizin wurden die Beziehungen verschiedener Hepatopathien mit klinischen Symptomen der Magenkrankheiten, des Magenchemismus und Ergebnissen der Gastroskopie verglichen ${ }^{1}$. Durch Einführung der Aspirationsbiopsie der Magenschleimhaut ${ }^{2,3}$ suchte man nach einer Korrelation zwischen morphologischen Veränderungen der Magenschleimhaut und chronischer Hepatitis und Lebercirrhose ${ }^{4-8}$.
Unsere Experimente verfolgten den Einfluss des Tetrachlormethan $\left(\mathrm{CCl}_{4}\right)$ auf morphologische und bio-

1 N. HeNNING, Die Entzindung des Magens (Leipzig 1934).

2 I. J. Wood, K. K. Ooro, R. MotteraM und A. Hughes, Lancet 19492,18 .

3 J. Tomenius, Gastroenterology 15, 498 (1950)

4 H. L. Stadel, K. Heinkel und G. Berg, Acta hepato-splenol. 10, 302 (1963).

5 K. Waldmann und J. A. Frisdor, Med. Klinik 55, 1190 (1960).

${ }^{B}$ E. D. Palmer, Am. J. dig. Dis. 18,323 (1951).

7 E. D. Palmer, Medicine 33, 199 (1954).

${ }^{B}$ R. A. Joske, E. S. FrnckF und I. J. Wood, Quant. J. Med. 24, 269 (1955). 